\title{
Stochastic Model for Profitability Evaluation of Demand Response by Electric Thermal Storage
}

\author{
Zane Broka, Karlis Baltputnis, Antans Sauhats \\ Institute of Power Engineering \\ Riga Technical University \\ Riga, Latvia \\ zane.broka@rtu.lv, karlis.baltputnis@rtu.lv, \\ sauhatas@eef.rtu.lv
}

\author{
Liga Sadovica, Gatis Junghans \\ Augstsprieguma tîkls, AS \\ Riga, Latvia \\ liga.sadovica@ast.lv, gatis.junghans@ast.lv
}

\begin{abstract}
In this paper, a tool for the economic assessment of a potential demand response asset used for power system balancing is presented. The model tackles uncertainties in electricity market prices and system imbalance by employing Monte Carlo simulations. While the model provides vast customizability options, the potential demand response benefits for a particular type of consumer, smart electric thermal storage, are the focus of the case study. It is found that such type of operations can be economically feasible for the asset owner, but on the condition that sufficient proportion of the balancing renumeration is shared with the owner by the aggregator.
\end{abstract} Carlo.

Keywords-aggregation; balancing; demand response; Monte

\section{INTRODUCTION}

Demand response (DR) is an increasingly enticing means the power system operators can employ in system control and management. There are several benefits DR can bring to the system, e.g., provision of ancillary services, contingency management, price volatility reduction, investment cost deferral etc. [1]. In principle, two main types of DR programs can be distinguished - price based (implicit), where the load follows some external price signal, and incentive based (explicit), where the DR asset owner is remunerated in either a classic direct control/interruptible load program or from an ancillary service/ capacity market [1].

To improve the energy independence and diversify the flexibility resources offered on the Baltic balancing market, the transmission system operators (TSOs) of the three Baltic states are planning to commence employment of DR assets for system balancing in the near future [2]. However, small loads do not have much influence on the overall system frequency, hence their control has to be aggregated to reach the required minimum balancing power bid size. This is usually done by an aggregator which is an entity that pools together the flexibility resources of several consumers and offers them to a marketplace or an operator directly [3].

On the one hand, for electricity end-users to become interested in DR provision, they need to be aware of the potential benefit they can gain. On the other hand, proper incentives need to be introduced for customers to participate in DR programs. However, the modeling of DR operation required for economic feasibility evaluation is quite complicated. An important issue

This work is part of the RealValue project. This project has received funding from the European Union's Horizon 2020 research and innovation programme under grant agreement No 646116. which should not be neglected is load recovery when consumers change their consumption patterns in the hours following a DR event [4]. Another peculiarity arises when modeling the flexibility potential of a consumer and uncertainties related to it [5]. Uncertainty is also a factor concerning the stochastic behavior of prices in electricity markets and system imbalances. To that end, Monte Carlo based simulations have proven to be an effective approach to handle modeling uncertainties [6].

In this study, Monte Carlo simulations are utilized in developing a software tool for DR economic potential that has been created in close collaboration with the national TSO. In essence, the tool establishes the potential economic benefit the owners of controllable load assets might achieve should they agree to participate in an explicit DR program, particularly, by having their load remotely reduced or increased to meet system balancing needs. The software has been developed using the MATLAB scripting environment [7].

This paper also provides a case study to evaluate the DR potential of a consumer who uses smart electric thermal storage devices for heating their dwelling [8].

\section{Methodology}

As stated previously, the main computational principle of this software lies in a Monte Carlo simulation-based approach for modeling the activations of DR and the related cash flows within a full year of the asset's operation. Consequently, the output of the model is in the form of probability distributions instead of one deterministic result as implying absolute certainty would be unreasonable when considering future processes. The tool is implemented via a number of software modules which are described in more detail in the following subsections.

\section{A. Input Module}

The overall setup of the model is based on the expected market conditions for DR operation in the Baltic states, since as of mid-2018 it is still under development. The input settings necessary to run the developed assessment model are primarily divided in four categories.

Firstly, the parameters which provide economic description of the DR asset and contractual conditions of its owner:

- asset service life (years); 
- capital expenditure (CAPEX) to enable participation in system balancing $(€)$, e.g., remote control hardware or software;

- annual fixed operating expenditure (F-OPEX) to maintain the DR provision ability $(€)$, e.g., additional bandwidth maintenance, related service fees etc.;

- variable operating expenditure (V-OPEX) arising from DR operations. This parameter can be modeled in three distinct ways $-€ / D R$ activation, $€ / D R$ affected load or $€ /$ unrecovered load;

- minimum balancing price for consumption reduction and maximum balancing price for consumption increase (either fixed $€ / \mathrm{MWh}$ or $\%$ from hourly day-ahead price) - parameters to establish the bid price limits of the DR asset's participation in the balancing market;

- a binary variable to establish if the owner of the DR asset itself is a balance responsible party (BRP) or not, which significantly changes how the cash flows are modeled;

- an option to select how the energy purchase price is modeled (only day-ahead $\Pi_{\mathrm{da}}^{t}$, day-ahead with markup in the form $k_{1} \cdot \Pi_{\mathrm{da}}^{t}+k_{2}$, fixed price derived from the simulated day-ahead price or scenario-independent general fixed price) $(€ / \mathrm{MWh})$;

- share of the TSO payment for load reduction which is passed on to the DR asset owner (\%); implying that the rest of the renumeration is received by the aggregator, BRP or other unspecified party.

Secondly, a technical description of the DR asset's hourly load and its flexibility has to be provided. This can be done either for a typical day or a typical week if applicable and with up to four distinct profiles to capture seasonality (i.e., the modeled year can be divided in four three-month periods).

As the DR activations are modeled with an hourly resolution, the most important parameters here are the maximum permitted number of DR events in a day or week, minimum time distance between any two DR events (hours), load flexibility direction for balancing (reduce, increase, both), minimum and maximum duration of a DR event (hours), maximum duration before load recovery (hours), load recovery factor (coefficient, where 1 implies that all the load reduced/increased during a DR event has to be recouped (increased/reduced) in the following hours. The meaning of these settings is better explained in Fig. 1, where green colors denote a DR event and red - the recovery.

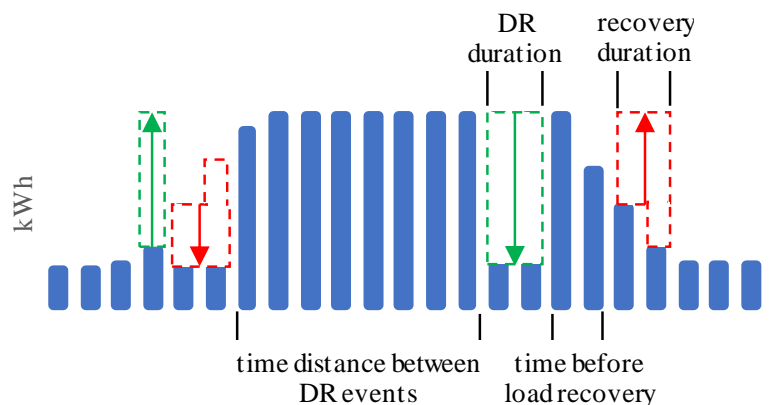

Fig. 1. Explanation of some of the DR modeling terms used
Note that the distance between two events is the time between the end of last recovery and beginning of the next DR activation. The area ratio between the green and red figures depends on the recovery factor, which can be selected different for the load increase and load reduction DR events. The hourly load profile with hourly upwards and downwards flexibility concludes the full technical description of the DR asset.

Thirdly, there are settings concerning the generation of dayahead price scenarios - expected mean price $(€ / \mathrm{MWh})$ for the normally distributed hours, expected maximum price $(€ / \mathrm{MWh})$ for the normally distributed hours, expected ratio between the mean weekday and holiday price, expected ratio between the mean day and night price (night defined as 22:00-6:00), expected minimum price $(€ / \mathrm{MWh})$. For each scenario these parameters are drawn from a normal distribution. Two more parameters ensure that the resulting price distributions more closely follow the skewness with right tail traditionally observed in electricity wholesale spot prices - percentage of hours where peaks outside the normal distribution occur and the expected maximum ( $€ / \mathrm{MWh})$ of such peaks. All the parameters described in this paragraph additionally have individually selectable standard deviations to ensure better controllability of the price scenario generation mechanism.

Finally, certain input parameters are needed to model the balancing market scenarios - expected balancing market liquidity (\% of hours when the TSO has imbalance it could cover with DR), upper and lower bounds of this parameter to ensure that in none of the scenarios the liquidity is drawn from outside this range, ratio of negative imbalance hours from all the hours with system balancing. The balancing price is drawn from the previously generated day-ahead price scenarios. The settings controlling this are - the expected ratio of hourly balancing price vs day-ahead price separately for negative and positive system imbalance, probability of extraordinarily high balancing price peaks and the maximum ceiling for the extraordinary balancing price (€/MWh).

\section{B. Day-Ahead Price Scenario Generation}

The input parameters described in the previous subsection are used to generate a pre-selected number of day-ahead price scenarios for a whole year with hourly resolution. The day-ahead price generation algorithm proceeds as follows.

1. From a normal distribution, draw price generation settings for each particular scenario (mean, min, max, ratios etc) using the expected values and standard deviations read from the input parameters.

2. For each scenario $s$, ensure that the drawn mean, min and max settings are not contradictory.

3. For each hour category within each scenario, calculate a coefficient necessary to enforce the weekday/holiday and day/night ratios as in (1) for weekday nights, (2) for weekday daytime, (3) for holiday nights and (4) for holiday daytime:

$$
k_{\mathrm{w}, \mathrm{n}}^{s}=\left(R_{\mathrm{w} / \mathrm{h}}^{s} \cdot \Pi_{\mathrm{da}}^{s, \text { avg }} /\left(2 / 7+5 / 7 \cdot R_{\mathrm{w} / \mathrm{h}}^{s}\right)\right) /\left(1 / 3+2 / 3 \cdot R_{\mathrm{d} / \mathrm{n}}^{s}\right) ;
$$




$$
\begin{gathered}
k_{\mathrm{w}, \mathrm{d}}^{s}=R_{\mathrm{d} / \mathrm{n}}^{s} \cdot\left(R_{\mathrm{w} / \mathrm{h}}^{s} \cdot \Pi_{\mathrm{da}}^{s, a v g} /\left(2 / 7+5 / 7 \cdot R_{\mathrm{w} / \mathrm{h}}^{s}\right)\right) /\left(1 / 3+2 / 3 \cdot R_{\mathrm{d} / \mathrm{n}}^{s}\right) ; \\
k_{\mathrm{h}, \mathrm{n}}^{s}=\left(\prod_{\mathrm{da}}^{s, \text { avg }} /\left(2 / 7+5 / 7 \cdot R_{\mathrm{w} / \mathrm{h}}^{s}\right)\right) /\left(1 / 3+2 / 3 \cdot R_{\mathrm{d} / \mathrm{n}}^{s}\right) \\
k_{\mathrm{h}, \mathrm{d}}^{s}=R_{\mathrm{d} / \mathrm{n}}^{s} \cdot\left(\Pi_{\mathrm{da}}^{s, \mathrm{avg}} /\left(2 / 7+5 / 7 \cdot R_{\mathrm{w} / \mathrm{h}}^{s}\right)\right) /\left(1 / 3+2 / 3 \cdot R_{\mathrm{d} / \mathrm{n}}^{s}\right)
\end{gathered}
$$

4. For each hour $t$ in each scenario $s$, generate day-ahead price as in (5) and (6), while ensuring they do not violate scenario minimum and maximum restrictions:

$$
\begin{gathered}
\Pi_{\mathrm{da}}^{s, t}=\max \left[N\left(k_{t}^{s},\left(\Pi_{\mathrm{da}}^{s, \mathrm{avg}}-\Pi_{\mathrm{da}}^{s, \min }\right) / 3\right), \Pi_{\mathrm{da}}^{s, \min }\right] ; \\
\Pi_{\mathrm{da}}^{s, t}=\min \left[\Pi_{\mathrm{da}}^{s, t}, \Pi_{\mathrm{da}}^{s, \text { norm } \max }\right] .
\end{gathered}
$$

5. Smoothen the generated time series with a moving average filter with a span of five elements.

6. Enforce the expected mean on the smoothened price:

$$
\Pi_{\mathrm{da}}^{s, t}=\Pi_{\mathrm{da}}^{s, t} \cdot \Pi_{\mathrm{da}}^{\text {exp,avg }} / \sum_{s=1}^{S}\left(\sum_{t=1}^{T} \Pi_{\mathrm{da}}^{s, t} /(S \cdot T)\right) .
$$

7. Finally, in each scenario, for $k_{\text {extra peak }}^{s} \%$ of hours add an increased price event:

$$
\Pi_{\mathrm{da}}^{s, t}=\Pi_{\mathrm{da}}^{s, t}+\Pi_{\mathrm{da}}^{\mathrm{s}, \text { extra max }}-\Pi_{\mathrm{da}}^{s, \text { norm max }}
$$

\section{Balancing Liquidity and Price Scenario Generation}

The balancing liquidity and price scenarios are generated as follows.

1. For each scenario, draw the balancing liquidity ( $\%$ of hours where TSO might request DR) parameter from a normal distribution.

2. Ensure that the drawn values respect the upper and lower bounds; if they do not, replace the value with the violated bound.

3. Since the model runs with hourly resolution, each hour with balancing liquidity has to be assigned either direction upwards or downwards balancing.

4. Generate upwards and downwards balancing prices for each hour in each scenario:

$$
\begin{gathered}
\Pi_{\text {bal, up }}^{s, t}=\Pi_{\mathrm{da}}^{s, t} \cdot \min \left(1, N\left(R_{\mathrm{up} / \mathrm{da}},\left(R_{\mathrm{up} / \mathrm{all}}-1\right) / 3\right)\right) ; \\
\Pi_{\text {bal, dwn }}^{s, t}=\Pi_{\mathrm{da}}^{s, t} \cdot \max \left(1, N\left(R_{\mathrm{dwn} / \mathrm{da}},\left(1-R_{\mathrm{dwn} / \mathrm{all}}\right) / 3\right)\right) .
\end{gathered}
$$

5. Combine the two timeseries for each scenario as per the hourly imbalance direction to obtain one balancing timeseries per scenario.

\section{Balancing Activation Simulation}

When all day-ahead electricity price and balancing scenarios have been generated, they can be paired, and the balancing activations can finally be estimated.

The purpose of this module is identifying the hours when the modeled DR asset can participate in balancing and when the energy recovery post-DR takes place. The program goes through each scenario sequentially checking each hour to test if activation conditions are met. The overall DR activation simulation algorithm is summarized in Fig. 2.

In the first conditional test block, all of these conditions have to be met:

- the minimum time distance since the previous DR activation is respected;

- the number of DR activations in the current day/week does not exceed the limit;

- there is demand for balancing in the system coinciding with the direction the DR asset owner is willing to provide services in (load reduction/increase);

- the DR asset has flexibility in the particular direction during the particular hour;

- the balancing price falls within the DR asset's bid limits;

- there is enough flexibility in the next hours for DR energy recovery respecting the max duration before load recovery constraint (relevant if the load recovery factor is nonzero).

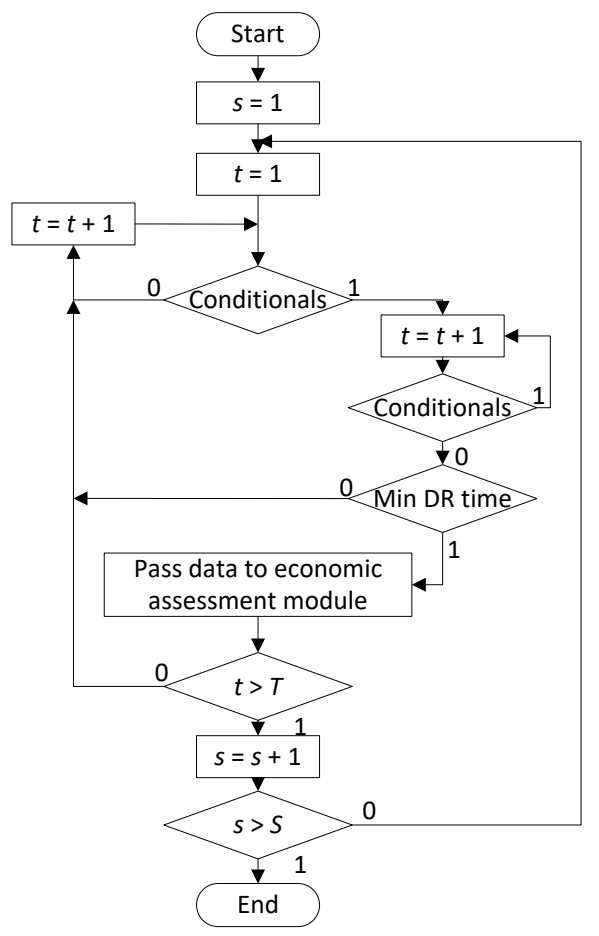

Fig. 2. Simplified viusalization of the DR activation simulation algorithm 
During the subloop with the second conditional block, it is tested if the duration of the DR event can be increased (the same conditions are checked with an additional test against the max duration of a DR event variable). Finally, it is checked whether the potential DR event duration meets the minimum limit.

Afterwards, if a DR event has been identified, information about it is passed on to the economic assessment module.

\section{E. Short-Term Economic Assessment}

The formulae (11)-(14) are used to calculate the cash flows associated with a simulated DR event. They depend on the direction of the DR induced load change and the balance responsibility status of the DR asset owner. The benefit is derived by contrasting the cash flows with and without DR. Beforehand, however, the energy purchase price timeseries are produced as mentioned in section II.A.

Variable $t D R$ denotes the set of hours when the DR event takes place and, consequently, trec denotes the set of hours when the recovery takes place. Since, theoretically, the DR event and recovery can span multiple hours, the multiplications in the following equations are implied to be matrix operations.

Benefit from load reduction if DR asset owner is BRP is composed from the income from the sold balancing energy (at balancing prices) and expenditure for recovery energy (at balancing prices):

$$
B_{\mathrm{BRP}}^{\mathrm{red}}=E_{\mathrm{DR}}^{s, t D R} \times \Pi_{\mathrm{bal}}^{s, t D R}-E_{\mathrm{rec}}^{s, t r e c} \times \Pi_{\mathrm{bal}}^{s, t r e c} .
$$

Benefit from load increase for a BRP depends on the expense for procured balancing energy during the DR event and income from sold balancing energy during the recovery:

$$
B_{\mathrm{BRP}}^{\text {incr. }}=-E_{\mathrm{DR}}^{s, t D R} \times \Pi_{\text {bal }}^{s, t D R}+E_{\text {rec }}^{s, \text { trec }} \times \Pi_{\text {bal }}^{s, \text { trec }} .
$$

For a DR asset owner who is not balance responsible, the benefit from load reduction derives from the income from sold balancing energy, savings from load reduction during the DR event (at retail purchase price since, unlike BRP, the owner has no obligation to balance their portfolio) and expense for recovery energy (at purchase price):

$$
B_{\mathrm{nonBRP}}^{\text {red. }}=E_{\mathrm{DR}}^{s, t D R} \times\left(\Pi_{\mathrm{bal}}^{s, t D R}+\Pi_{\mathrm{pp}}^{s, t D R}\right)-E_{\mathrm{rec}}^{s, t r e c} \times \Pi_{\mathrm{pp}}^{s, t r e c} .
$$

Finally, for a non-BRP, the load increase cash flow components are the expense for procured balancing energy (at balancing price) and the savings from load reduction in the recovery phase (at purchase price):

$$
B_{\text {nonBRP }}^{\text {incr. }}=-E_{\mathrm{DR}}^{s, t D R} \times \Pi_{\mathrm{bal}}^{s, t D R}+E_{\mathrm{rec}}^{s, \text { trec }} \times \Pi_{\mathrm{pp}}^{s, \text { trec }} .
$$

The benefit from load reduction and/or increase is contrasted to the fixed and variable OPEX to find the overall benefit from participation in DR in each scenario throughout the whole year.

\section{F. Long-Term Economic Assessment}

The modeling outcome from the one-year run is extrapolated to further years for the whole service life of the DR asset by applying the previously selected discount rate. Several widely used investment assessment metrics are now calculated, such as net present value (NPV), internal rate of return (IRR) and payback period (PP). Once the long-term assessment is finalized, the calculation results are summarized and output to figures and data tables.

\section{CASE STUDY}

\section{A. Assumptions}

The case study aims to apply our developed software tool for economic assessment of smart electric thermal storage (SETS) participation in DR. The subject of the study is a hypothetical household having five SETS devices at their disposal with $2.2 \mathrm{~kW}$ input power and $15.4 \mathrm{kWh}$ storage capacity each. The owner is not balance responsible and is willing to participate in both upward and downward DR (which requires the SETS equipment to never be disconnected from the outlet and the gateway). We assume the asset service life to be 15 years, discount rate $-3 \%$, CAPEX - $200 €$ (to cover gateway costs) and annual F-OPEX - $20 €$ (service and other related costs). For simplicity sake, it is implied for now the householder purchases electricity at wholesale price. It is also assumed that the aggregator passes on to the DR asset owner all of the TSO payments for load reduction (however, the effect of this assumption will be explicitly addressed).

In regards to the load profile and flexibility, we set a maximum number of 14 DR activations per week, but do not restrict the time between them. In this study, we do not allow for multi-hour DR events. Maximum duration before load recovery is set to 12 hours and the recovery factor is set to 0.9 both for load reduction and increase (this implies some energy savings in case of load reduction and some wasted energy in case of increase).

The seasonal heating demand data is derived from the model of [9], where it was seen that the overall heating demand in summer, spring and autumn is approximately $10 \%, 50 \%$ and $20 \%$ of the winter demand respectively. Consequently, we assume that, in summer, there is one SETS unit that charges 2..5 hours a day, can be disconnected anytime during the charging and another unit can be turned on whenever necessary.

In autumn, one SETS unit charges for the full seven hours, but can be disconnected at request; the other remaining units can be switched to charging when necessary. In winter, four units are in full operation; in spring - two, in either case the operational units can be switched off and any idle units - set to charging.

The day-ahead price scenario generation settings are derived from an analysis of the Nord Pool Latvian bidding area prices during the period of 01.06.2017-31.05.2018. Expected mean price for $99.5 \%$ of hours is $37.75 € / \mathrm{MWh}$, expected maximum price for $99.5 \%$ of hours - $119.5 € / \mathrm{MWh}$, expected ratio between mean weekday and holiday prices -1.25 , expected ratio between mean daytime and nighttime prices -1.44 , expected 
minimum price $-1.59 € / \mathrm{MWh}$, expected rare maximum $255 € / \mathrm{MWh}$. A total of 1000 price scenarios are generated.

The balancing scenario settings are derived from the common Baltic balancing market launched on 01.01.2018. The expected balancing market liquidity is $64.97 \%$, ratio of negative vs positive imbalance hours -0.44 , expected balancing price during positive system imbalance $-0.58 \mathrm{pu}$ from the day-ahead price, expected balancing price during negative system imbalance - $1.49 \mathrm{pu}$ from the day-ahead price. Zero extraordinary balancing price events are assumed.

The generated hourly day-ahead market and balancing prices across the thousand scenarios are summarized in Fig. 3.

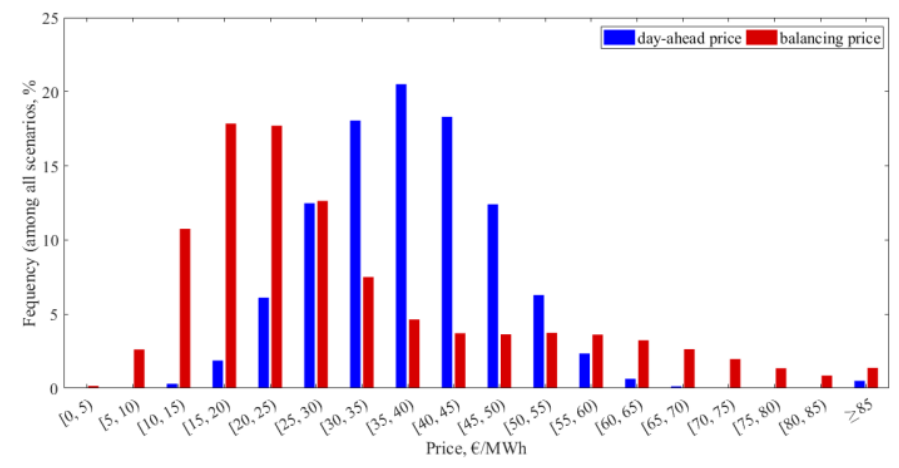

Fig. 3. Day-ahead (blue) and balancing (red) price histograms

\section{B. Results}

Though the simulation results imply there have been much more DR activations for load increase than for load reduction (on average, 452 times for increase and 199 for reduction), Fig. 4 suggests that the reduction operations have been overall more economically beneficial (scenario average of $46.50 €$ vs $12.71 €)$. This is also reflected in the specific benefit per DR activation (mere $3.92 € / \mathrm{MWh}$ average for increase, but $49.80 € / \mathrm{MWh}$ for reduction). This can primarily be explained by two factors, the additional positive cash flow component in case of load reduction (see Fig. 4) and the initially assumed load recovery factor 0.9 for both directions, which implied that load increase DR is slightly wasteful in terms of energy consumption.

The average NPV is at $268.10 €$, however, Fig. 5 and Fig. 6 show that there are some scenarios $(3.6 \%)$ where the NPV is still negative at the end of the selected service life. The average IRR is $17.56 \%$. The average payback period is thus 7.23 years while the median is 6 years, which signals that the outlier scenarios are likely skewing the mean. Indeed, Fig. 5 shows that some of the outlier scenarios have not reached payback even by year 20 .

Nevertheless, while an expectable $268.10 €$ benefit accumulated during a 15-year period is not necessarily very enticing for a whole household to allow remote control of their heating equipment, this result does serve as valuable first insights in the assessment of the economical potential of participation in explicit DR on a dwelling level.

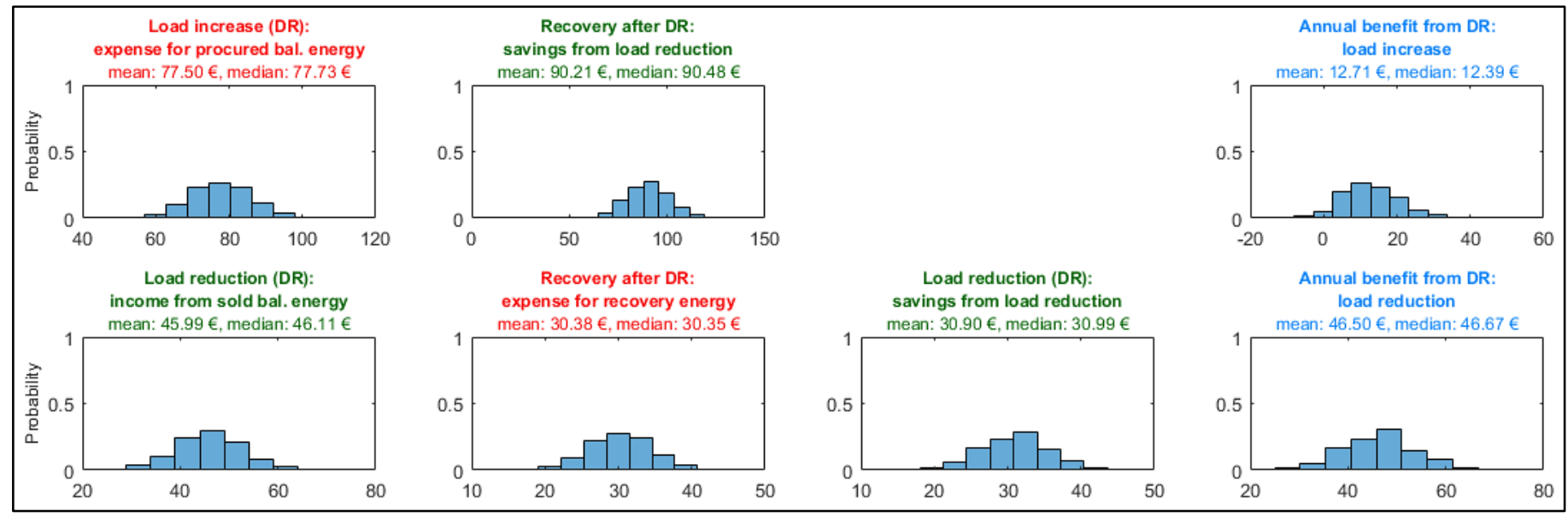

Fig. 4. Economic assessment for a single modeled year
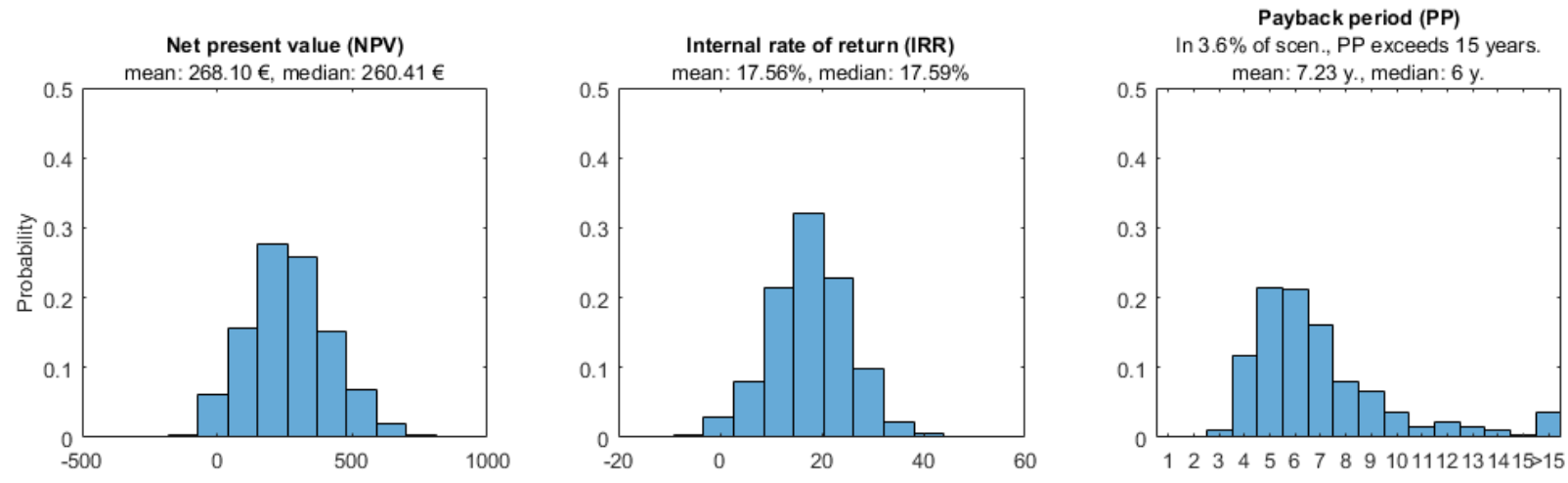

Fig. 5. Long-term economic assessment for the asset service life (15 years) 


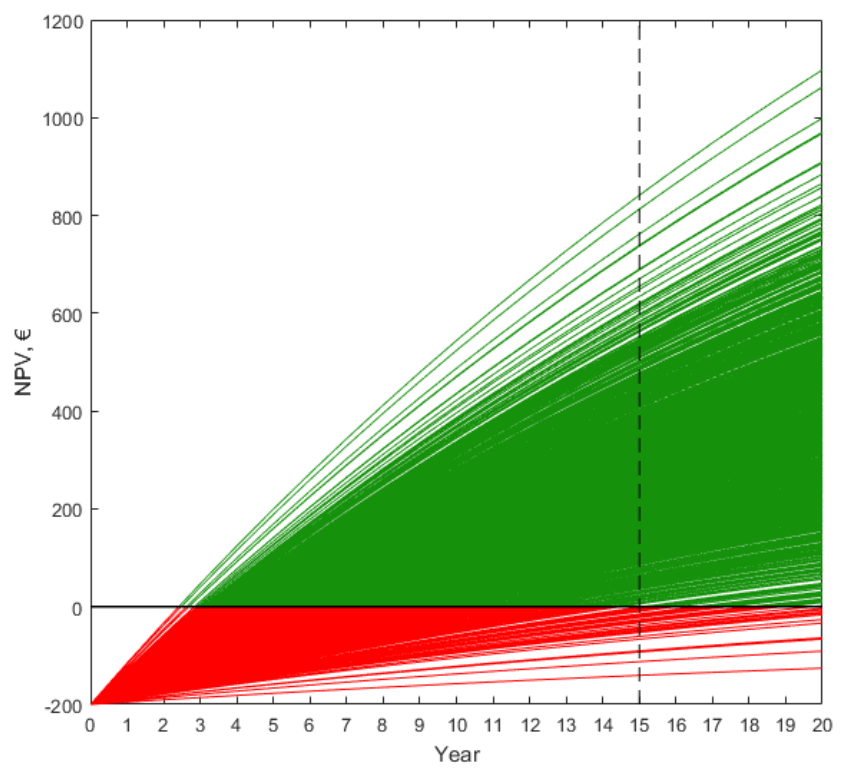

Fig. 6. The cumulative cash flows in the simulated scenarios

A note should be made, however, that the initial assumption of a household in Latvia exclusively heated by SETS devices is not strictly realistic since even conventional electric heating which could be replaced is not currently widespread in Latvia and SETS is on a significantly higher price range than conventional heaters. If the SETS device costs where also included in DR CAPEX calculations, payback would not be possible.

\section{Impact of the Payment Sharing on SETS DR Feasibility}

Additionally, the assumption that $100 \%$ of the TSO payment for load reduction is received by the DR asset owner is objectionable. To alleviate this limitation of the study, we completed several additional model runs with all the same input data only varying the share coefficient. The results from the repeated runs are summarized in the following table. Evidently, the share of TSO payment the DR asset owner receives has to be higher than $50 \%$ for the participation in an explicit DR program to be economically meaningful.

TABLE I. EFFECT OF BENEFIT SHARING ON DR FEASIBILITY

\begin{tabular}{|l|c|c|c|c|}
\hline \multirow{2}{*}{$\begin{array}{c}\text { TSO payment } \\
\text { share passed to } \\
\text { the DR asset } \\
\text { owner }\end{array}$} & NPV, $\boldsymbol{\epsilon}$ & IRR, \% & $\begin{array}{c}\text { PP, years } \\
\text { mean / med. }\end{array}$ & $\begin{array}{c}\text { \%of } \\
\text { scenarios } \\
\text { where PP } \\
\text { impossible }\end{array}$ \\
\cline { 2 - 5 } & 268.10 & 17.56 & $7.23 / 6$ & 0.0 \\
\hline $100 \%$ & 209.37 & 14.57 & $8.51 / 7$ & 0.0 \\
\hline $80 \%$ & 149.28 & 11.32 & $11.17 / 8$ & 0.0 \\
\hline $70 \%$ & 101.08 & 8.57 & $15.06 / 10$ & 0.7 \\
\hline $60 \%$ & 35.43 & 4.37 & $22.92 / 13$ & 2.0 \\
\hline $50 \%$ & -6.85 & 1.47 & $29.99 / 16$ & 3.4 \\
\hline
\end{tabular}

\section{CONCLUSIONS}

The developed Monte Carlo simulation-based DR economic assessment tool has proven to be useful in providing preliminary evaluation of the potential benefits controllable load asset owner might gain by participating in the power system balancing via an explicit DR program. However, the model employed requires quite detailed knowledge of the technical characteristics of the DR asset, especially in regard to its available flexibility with an hourly resolution. In general, the results are assumption-sensitive, thus any output should not be viewed independently of the input.

The preliminary results signal that electric thermal storage devices can recoup the additional investments necessary to make them DR-ready, but only if more than $50 \%$ of the load reduction renumeration is passed on to the asset owner. In fact, the stochastic output of the model shows that even at $100 \%$ renumeration, there is a small probability that the payback period could exceed the asset service life. Realistically, however, such a full payment sharing is unlikely as the aggregation service provider also needs incentives for its operation.

In conclusion, a more accurate DR economic feasibility assessment would require near perfect beforehand knowledge of the contractual setup between the DR asset owner, aggregator, BRP, TSO and other potentially linked parties. However, the current version of the tool already allows modeling a variety of different setups which enables studies on finding the most suitable business case for a particular application. Nevertheless, further improvements of the tool and subsequent more rigorous validation are in the plans.

\section{REFERENCES}

[1] M. H. Albadi and E. F. El-Saadany, "A summary of demand response in electricity markets,” Electr. Power Syst. Res., vol. 78, no. 11, pp. 1989-1996, 2008.

[2] Augstsprieguma tīkls AS, Elering AS, and Litgrid UAB, "Demand response through aggregation - a harmonized approach in Baltic region," 2017.

[3] L. Gkatzikis, I. Koutsopoulos, and T. Salonidis, "The role of aggregators in smart grid demand response markets," IEEE J. Sel. Areas Commun., vol. 31, no. 7, pp. 1247-1257, 2013.

[4] D. T. Nguyen, M. Negnevitsky, and M. De Groot, "Modeling load recovery impact for demand response applications," IEEE Trans. Power Syst., vol. 28, no. 2, pp. 1216-1225, 2013.

[5] M. Vallés, A. Bello, J. Reneses, and P. Frías, "Probabilistic characterization of electricity consumer responsiveness to economic incentives," Appl. Energy, vol. 216, pp. 296-310, 2018.

[6] H. Janssen, "Monte-Carlo based uncertainty analysis: Sampling efficiency and sampling convergence," Reliab. Eng. Syst. Saf., vol. 109, pp. 123-132, 2013.

[7] “MATLAB." The MathWorks Inc, Natick, Massachusetts.

[8] Z. Broka, J. Kozadajevs, A. Sauhats, D. P. Finn, and W. J. N. Turner, "Modelling residential heat demand supplied by a local smart electric thermal storage system," in 2016 57th International Scientific Conference on Power and Electrical Engineering of Riga Technical University (RTUCON), 2016, pp. 1-8.

[9] L. Petrichenko, Z. Broka, and A. Sauhats, "Impact of smart electric thermal storage on distribution grid," Conf. Proc. - 2017 17th IEEE Int. Conf. Environ. Electr. Eng. 2017 1st IEEE Ind. Commer. Power Syst. Eur. EEEIC / I CPS Eur. 2017, 2017. 